\title{
Impact of examined lymph node count on staging and long-term survival of patients with node-negative stage III gastric cancer: a retrospective study using a Chinese multi-institutional registry with Surveillance, Epidemiology, and End Results (SEER) data validation
}

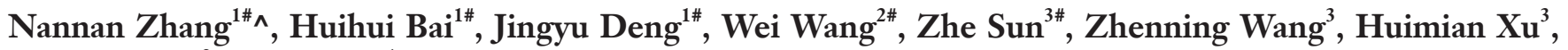 \\ Zhiwei Zhou' ${ }^{2}$ Han Liang'
}

${ }^{1}$ Department of Gastric Cancer, Tianjin Medical University Cancer Institute \& Hospital, National Clinical Research Center of Cancer, Key Laboratory of Cancer Prevention and Therapy, Tianjin's Clinical Research Cancer for Cancer, Tianjin, China; ${ }^{2}$ Department of Gastric and Pancreatic Surgery, Sun Yat-sen University Cancer Center, State Key Laboratory of Oncology in South China, Collaborative Innovation Center for Cancer Medicine, Guangzhou, China; ${ }^{3}$ Department of Surgical Oncology, the First Affiliated Hospital of China Medical University, Shenyang, China

Contributions: (I) Conception and design: J Deng, N Zhang, H Bai; (II) Administrative support: J Deng, H Liang, H Xu, Z Zhou; (III) Provision of study materials or patients: J Deng, N Zhang, H Bai, W Wang, Z Sun, Z Wang; (IV) Collection and assembly of data: N Zhang, H Bai, J Deng; (V) Data analysis and interpretation: N Zhang, H Bai, J Deng; (VI) Manuscript writing: All authors; (VII) Final approval of manuscript: All authors.

\#These authors contributed equally to this work.

Correspondence to: Jingyu Deng, MD. Department of Gastric Cancer, Tianjin Medical University Cancer Institute \& Hospital, National Clinical Research Center of Cancer, Key Laboratory of Cancer Prevention and Therapy, Tianjin's Clinical Research Cancer for Cancer, Tianjin, China. Email: dengery@126.com; Han Liang, MD. Department of Gastric Cancer, Tianjin Medical University Cancer Institute \& Hospital, National Clinical Research Center of Cancer, Key Laboratory of Cancer Prevention and Therapy, Tianjin's Clinical Research Cancer for Cancer, Tianjin, China. Email: tjlianghan@126.com; Zhiwei Zhou, MD. Department of Gastric and Pancreatic Surgery, Sun Yat-sen University Cancer Center, State Key Laboratory of Oncology in South China, Collaborative Innovation Center for Cancer Medicine, Guangzhou, China. Email: zhouzhw@sysucc.org.cn; Huimian Xu, MD. Department of Surgical Oncology, the First Affiliated Hospital of China Medical University, Shenyang, China. Email: xuhuimian@126.com.

Background: Accumulating evidence has confirmed the potential prognostic value of examined lymph nodes (ELNs) in patients with gastric cancer (GC). However, there is currently no consensus on the threshold ELN number for predicting both stage migration and long-term survival, especially in patients with stage III GC. This study aimed to validate the need to increase the ELN count to improve its prognostic accuracy in node-negative patients with stage III GC after curative gastrectomy.

Methods: This retrospective, population-based study analyzed the clinical data of 84 patients with nodenegative stage III GC from three high-volume institutions in China and 196 cases from the Surveillance, Epidemiology and End Results (SEER) program registry. The optimal number of ELNs was determined by receiver operating characteristic (ROC) curve analysis. Clinicopathological characteristics significantly related to survival were evaluated using the Kaplan-Meier method and Cox proportional hazards analysis. Stratified analyses were adopted to assess the prognostic predictive ability of the identified optimal number of ELNs in different populations. Survival differences among subgroups were analyzed to assess the impact of ELN count on stage migration according to overall survival (OS) among GC patients.

Results: The optimal number of ELNs was $>31$ according to ROC analysis of patients with node-negative stage III GC who underwent gastrectomy. Multivariate analysis identified ELNs as an independent predictor

^ ORCID: 0000-0003-0582-8934. 
of postoperative OS in patients with node-negative stage III GC in both the Chinese cohort [hazard ratio (HR) $0.235 ; \mathrm{P}<0.001$ ] and the SEER cohort (HR 0.421; $\mathrm{P}<0.010)$. Stratified analysis demonstrated that $>31$ ELNs was a prerequisite for accurate prognostic evaluation of patients with node-negative stage III GC, regardless of sex, tumor size, and other factors. Stage migration between pT4bN0M0 and pT4bN1M0 was detected in patients with > 31 ELNs. A nomogram was created to predict OS among patients with node-negative stage III GC. These results were validated using data from the SEER cohort.

Conclusions: The number of ELNs was significantly associated with prognosis in patients with stage III GC after gastrectomy with systemic lymphadenectomy in both the Chinese and SEER cohorts. The results suggest that $>31$ ELNs are required for an accurate prognostic evaluation in patients with GC, especially those with node-negative stage III GC.

Keywords: Stomach; neoplasm; lymph node; metastasis; prognosis

Submitted Feb 05, 2020. Accepted for publication Jul 20, 2020.

doi: $10.21037 /$ atm-20-1358a

View this article at: http://dx.doi.org/10.21037/atm-20-1358a

\section{Introduction}

Gastric cancer (GC) is the second-leading cause of cancerrelated death, with the highest mortality rates occurring in East Asia (1). Recent advances in screening systems and endoscopic instruments have allowed diagnosis of more patients at an early stage, and minimally invasive treatment administered to patients with early GC can yield a favorable prognosis $(2,3)$. However, many patients still have advanced GC at the time of diagnosis, and despite advances in treatment strategies, the prognosis for stage III GC remains poor (4). We therefore focused on stage III GC, which accounts for approximately $50 \%$ of all cases of GC diagnosed in China.

The best treatment for advanced GC is generally considered to be radical gastrectomy with systematic lymph node $(\mathrm{LN})$ dissection and adjuvant therapy $(5,6)$. $\mathrm{LN}$ involvement is one of the most important features determining the adjuvant treatment strategy and the long-term survival of patients with resectable GC. LN management is also a key factor in assuring precise nodal staging, both by identifying $\mathrm{LN}$ involvement and determining the extent of disease and in terms of the therapeutic effect of clearing potential LN metastatic (LNM) lesions. The number of examined LNs (ELNs) is significantly associated with LNM, and a higher number of ELNs is generally considered to indicate a higher number of LNMs (7-9). Previous studies also showed that node-negative cohorts with fewer ELNs may include some patients with node-positive disease $(10,11)$. A higher number of ELNs was associated with more accurate staging and stage migration, and improved harvesting of LNs may lead to reclassification of some individuals from nodenegative to node-positive $(12,13)$.

In light of these considerations, we aimed to analyze the associations between ELN number and long-term survival among patients with resected node-negative stage III GC. We present the following article in accordance with the STROBE reporting checklist (available at http://dx.doi. org/10.21037/atm-20-1358a) (14).

\section{Methods}

\section{Patients}

\section{Chinese multi-institutional data}

We retrospectively collected the clinical data of 8,884 patients who underwent gastrectomy for GC at three Chinese institutions between January 1, 2000 and December 31, 2011. The inclusion criteria were as follows: (I) histological diagnosis of primary cancer of the stomach; (II) no history of gastrectomy or other malignancy; (III) no non-curative surgical factors (such as distant metastasis, positive peritoneal cytology, or peritoneal dissemination); (IV) no esophagogastric junction tumor; (V) pathologically negative resection margins (R0 resection); (VI) underwent D2 or D2 plus lymphadenectomy according to Japanese Gastric Cancer Treatment Guideline 2010, Version 3; (VII) patients who remained alive during the initial hospital stay and the first postoperative month; (VIII) no preoperative treatment; and (IX) pathological stage III GC according to the eighth American Joint Committee on Cancer (AJCC) 
TNM classification. Patients with gastric stump cancer and patients with missing or insufficient data for ELNs, pathological findings, or macroscopic tumor size were excluded. All procedures were conducted in accordance with the Declaration of Helsinki (as revised in 2013) (15).

Ethical approval was obtained from the institutional review board of the Tianjin Medical University Cancer Institute \& Hospital (No: bc2018037). Primary tumors were resected en bloc via lymphadenectomy, in accordance with the guidelines of the Japanese Gastric Cancer Association (16), with surgical procedures mainly carried out in accordance with the Japanese Gastric Cancer Treatment Guidelines (17). Surgical experts who routinely carry out standard gastrectomy at each of the medical centers performed all the surgeries. LNs were harvested during surgical resection of GC and examined postoperatively by pathologists. ELN counts in the registry were generated by adding the surgeons' intraoperative harvested LN count to the number of LNs identified postoperatively by pathologists. All enrolled patients were staged according to the TNM classification for GC (eighth edition). Moreover, all specimens were analyzed by two professional pathologists in each institution, and different opinions were resolved through discussion to establish the ultimate diagnosis results (18).

All patients received standard follow-up, including laboratory and clinical examinations after discharge from the hospital every 3 months for the first 3 years, every 6 months during the fourth and fifth years, and once a year thereafter until the patient died or until the date of last follow-up (December 2016). Three doctors in each medical center are responsible for follow-up and recording of patients' information.

\section{Surveillance, Epidemiology, and End Results (SEER) database}

The SEER cohort was derived from the SEER database (November 2016 submission) using SEER*Stat software (National Cancer Institute). All patients with GC who underwent gastrectomy from 2004 to 2011 were identified (19). Patients with incomplete or missing information on depth of invasion, LN status, or status of distant metastasis were excluded. Patients were uniformly reviewed and restaged according to the eighth edition of the TNM classification.

\section{Stage migration}

Nodal stage migration refers to the change in stage distribution in a particular cancer population, possibly as a result of using a more sensitive method for detecting involved LNs (20). In this study, stage migration was defined as: (I) no statistically significant survival differences detected in several subgroups of patients with insufficient ELNs in the specific pTNM stages; (II) statistically significant survival differences detected in several subgroups of patients with sufficient ELNs in the specific pTNM stages.

\section{Statistical analysis}

The primary endpoint of this study was evaluation of the prognostic significance of the number of ELNs in patients with node-negative pathological stage III GC who underwent curative resection. The secondary endpoints were determination of the optimal cut-off value for the number of ELNs for predicting prognosis, and its significance in various subgroups. The optimal cut-off value of ELNs to predict 5-year postoperative survival was determined via receiver operating characteristic curve (ROC) analysis. Clinicopathological characteristics significantly related to patient survival were evaluated using the Kaplan-Meier method and Cox proportional hazards analysis. Stratified analyses were used to evaluate the prognostic predictive ability of the optimal number of ELNs in different subgroups, and to demonstrate stage migration in accordance with the different ELN values. A nomogram was further applied to predict overall survival (OS) of patients with node-negative stage III GC based on the predictive model. Details of GC cases in the SEER database were extracted using SEER*Stat version 8.3.6. Statistical analyses were performed using SPSS version 22 (SPSS Inc., Chicago, IL, USA), R version 3.6.1, and GraphPad Prism 8.0.2. All tests were two-sided and $\mathrm{P}<0.05$ was considered statistically significant.

\section{Results}

\section{Patients clinicopathological features and optimal ELN cut-off}

Regarding patients from the Chinese institutions, we collected the clinical data of 8,884 patients who underwent gastrectomy for GC. After applying the inclusion and exclusion criteria, 2,942 patients with stage III GC were analyzed and 84 node-negative patients from the Chinese database were considered eligible (median age, 59 years; range, 34-79 years) (Figure 1A). Regarding the SEER 

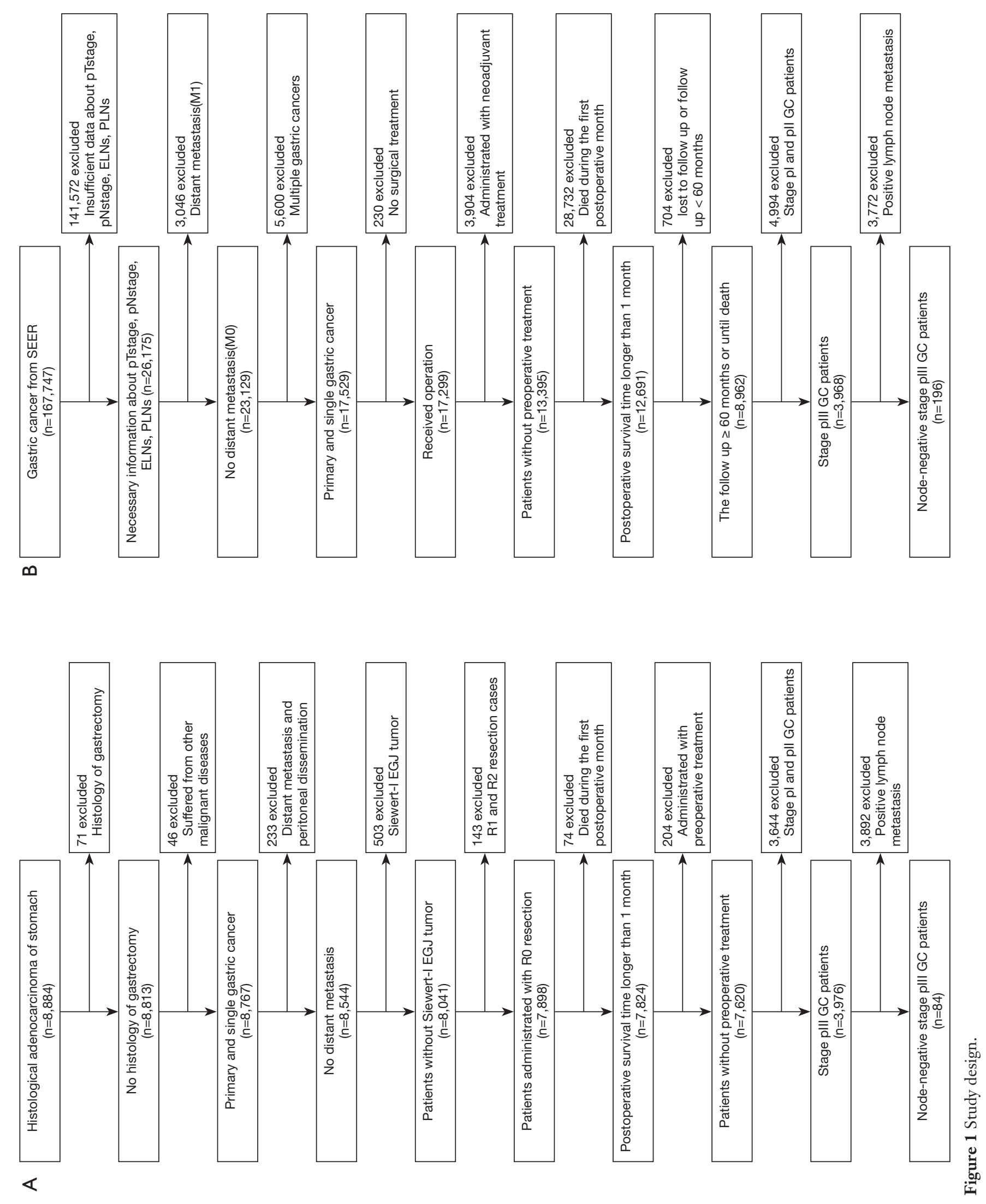

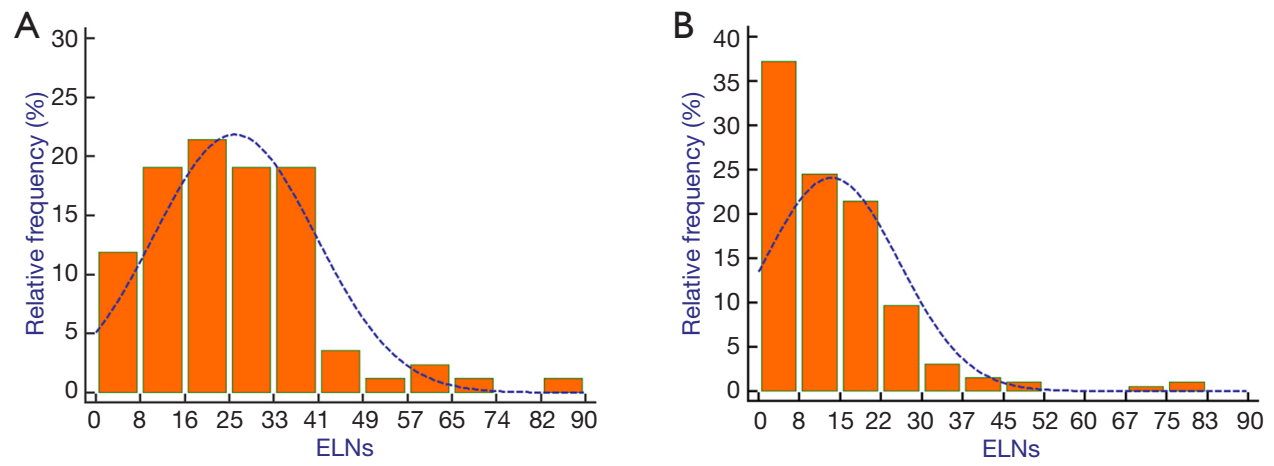

Figure 2 Distribution of the number of examined lymph nodes in the China registry and the SEER database. ELNs, examined lymph nodes; SEER, Surveillance, Epidemiology, and End Results.

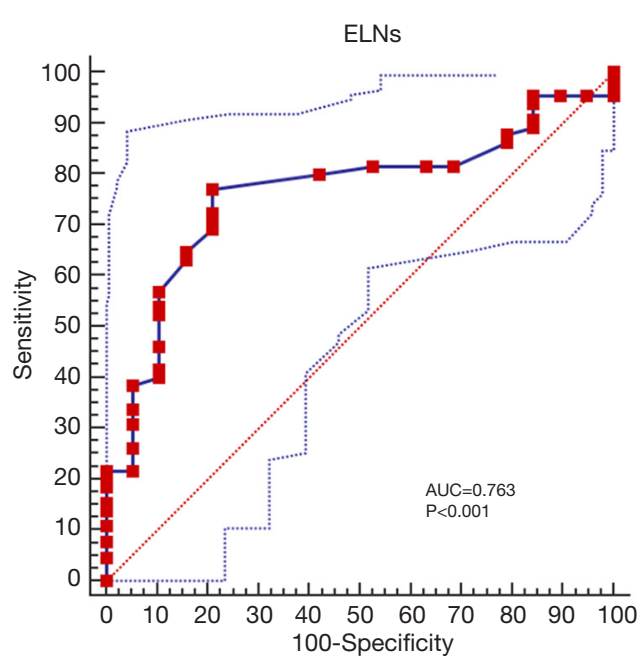

Figure 3 Receiver operating characteristic (ROC) curve analysis of the numbers of ELNs associated with 5-year mortality after surgery in node-negative stage III patients. ELNs, examined lymph nodes; SEER, Surveillance, Epidemiology, and End Results.

database, a total of 167,747 patients were initially included. After excluding patients with missing information, data for 3,968 stage pIII GC patients were analyzed, and 196 nodenegative patients from 18 SEER registries were eligible (median age, 69 years; range, 33-92 years) (Figure 1B). The median follow-up was 27 months for Chinese database (range, 2-126 months) and 25 months for SEER database (range, 2-147). The distribution of the number of ELNs differed between the two cohorts (Figure 2). The Chinese cohort had a larger number of ELNs (median, 24; range, 3-82) than the SEER cohort (median, 12; range, 1-79). Therefore, the Chinese database was defined as the training cohort and the SEER database as the external validation cohort.
ROC analysis of patients in the Chinese cohort who underwent gastrectomy for stage III node-negative GC revealed that $>31$ ELNs had the largest influence on prognosis 5 years after surgery (Figure 3), and 31 was therefore set as the optimum discriminative cut-off for the number of ELNs. The patients in the Chinese and SEER cohorts were classified into groups with $\leq 31$ ELNs ( $\mathrm{n}=55$ and 180, respectively) and $>31$ ELNs ( $\mathrm{n}=29$ and 16, respectively) according to the optimal ELN cut-off.

\section{Survival analyses of patients with node-negative stage III GC}

In this retrospective, population-based study, we used the SEER cohort to validate the association between the number of ELNs and OS determined in the Chinese cohort. Univariate analysis identified age, tumor size, Lauren classification, type of gastrectomy, and ELN clinicopathological characteristics as significantly associated with OS after curative surgery in the 84 node-negative patients in the Chinese cohort (Table 1, Figure 4). Age and ELNs were also significantly associated with OS in the 196 node-negative patients in the SEER cohort (Table 2, Figure 5). All five characteristics were included in a multivariate Cox proportional hazards model (forward stepwise procedure) to adjust for the effects of covariates in the Chinese cohort. ELNs [hazard ratio (HR) 0.235; $\mathrm{P}<0.001$ ], age (HR 1.720; $\mathrm{P}=0.049)$, tumor size (HR 2.012; $\mathrm{P}=0.034$ ), and type of gastrectomy (HR 0.699; $\mathrm{P}=0.042$ ) were identified as independent predictors for OS in postoperative patients with node-negative stage III GC (Table 1). Age, ELNs, tumor size, and Lauren classification were included in the multivariate analysis in the SEER cohort, and ELNs (HR 0.421; $\mathrm{P}<0.010$ ) and age (HR 1.584; $\mathrm{P}=0.008$ ) were identified as independent predictors for OS in postoperative node-negative patients (Table 2). 
Table 1 Clinicopathologic variables and univariate analysis of the Chinese training cohort of node-negative stage III gastric cancer patients

\begin{tabular}{|c|c|c|c|c|c|c|}
\hline Population & $\begin{array}{c}\text { No. of } \\
\text { patients }\end{array}$ & $\begin{array}{c}\text { 3-year OS } \\
(\%)\end{array}$ & $\begin{array}{c}\text { 5-year OS } \\
(\%)\end{array}$ & $\begin{array}{c}\text { Univariate analysis } \\
\mathrm{P} \text { value }\end{array}$ & \multicolumn{2}{|c|}{ Multivariate analysis } \\
\hline Gender & & & & 0.456 & & \\
\hline Female & 18 & 27.8 & 16.8 & & & \\
\hline Male & 66 & 40.9 & 22.7 & & & \\
\hline$\leq 60$ & 40 & 50 & 27.5 & & 1 (Ref.) & \\
\hline$>60$ & 44 & 27.3 & 15.9 & & $1.720(1.013-2.961)$ & \\
\hline Tumor size, $\mathrm{cm}$ & & & & 0.006 & & 0.034 \\
\hline$\leq 4$ & 19 & 63.2 & 47.7 & & 1 (Ref.) & \\
\hline Upper third & 26 & 38.5 & 23.2 & & & \\
\hline Middle third & 16 & 31.3 & 18.8 & & & \\
\hline Lower third & 29 & 44.8 & 24.1 & & & \\
\hline$>2 / 3$ stomach & 13 & 30.8 & 15.4 & & & \\
\hline Lauren classification & & & & 0.034 & & 0.265 \\
\hline Intestinal & 43 & 44.2 & 30.2 & & 1 (Ref.) & \\
\hline Diffuse & 41 & 31.7 & 12.2 & & $1.344(0.800-2.258)$ & \\
\hline Type of gastrectomy & & & & 0.016 & & 0.042 \\
\hline ELNs $>31$ & 29 & 69.0 & 51.7 & & $0.235(0.125-0.444)$ & \\
\hline
\end{tabular}

No., number; OS, overall survival; HR, hazard ratio; 95\% CI, 95\% confidence interval; Ref, reference; TG, total gastrectomy; PG, proximal gastrectomy; DG, distal gastrectomy; ELNs, examined lymph nodes.

\section{Subgroup analyses of node-negative patients}

We carried out subgroup analyses using the Kaplan-Meier method to clarify the prognostic predictive ability and clinical significance of the number of ELNs in patients with node-negative stage III GC (Tables 3,4). We compared the prognoses between patients with $\leq 31$ and $>31$ ELNs among patients stratified by sex, age at surgery, tumor size, tumor location, Lauren classification, and type of gastrectomy in the Chinese cohort. Patients with $>31$ ELNs had significantly better OS rates (e.g., 3- and 5-year OS) and higher mortality (HR 0.235, $\mathrm{P}<0.001$ ) compared with patients with $\leq 31$ ELNs. Similar findings were observed in patients stratified as follows: female (HR 8.255, $\mathrm{P}=0.054$ ), male (HR 3.020, $\mathrm{P}=0.001$ ), age $\leq 60$ years ( $\mathrm{HR} 4.616$, $\mathrm{P}=0.001)$, age $>60$ years (HR 3.653, $\mathrm{P}=0.002)$, tumor $\leq 4 \mathrm{~cm}$ (HR 10.849, $\mathrm{P}=0.001)$, tumor $>4 \mathrm{~cm}(\mathrm{HR} 2.795, \mathrm{P}<0.001)$, upper third (HR 9.966, $\mathrm{P}=0.036$ ), lower third (HR 5.571, $\mathrm{P}<0.001$ ), intestinal GC (HR 2.784, $\mathrm{P}=0.005)$, diffuse GC (HR 3.952, $\mathrm{P}=0.003)$, total gastrectomy (TG) (HR 3.376, $\mathrm{P}=0.013)$, and distal gastrectomy (DG) $(\mathrm{HR} 5.597, \mathrm{P}<0.001)$ 
A

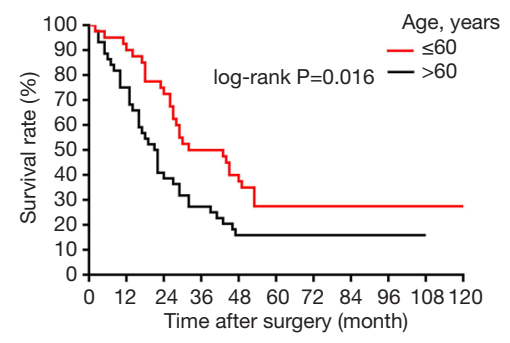

C

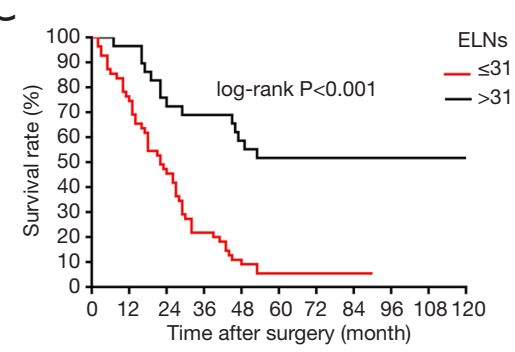

B
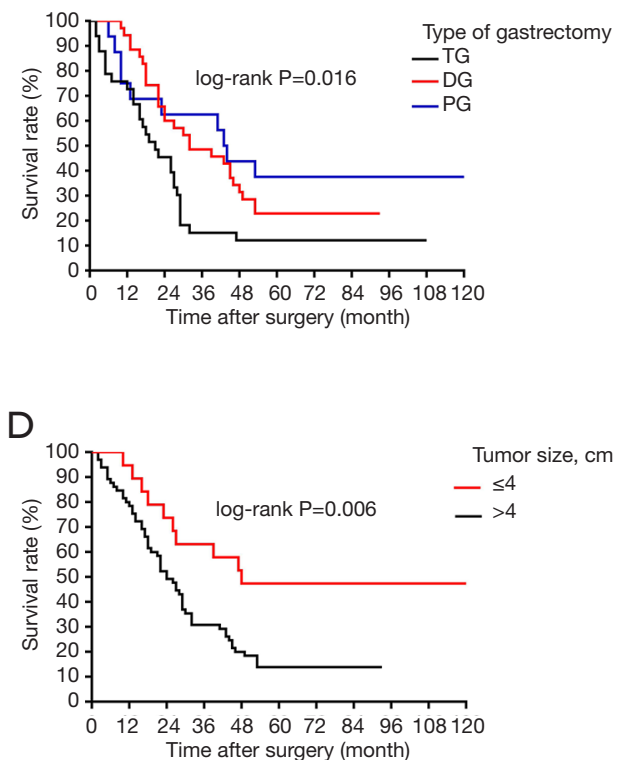

Figure 4 Survival curves of patients in Chinese training cohort according to subgroups: (A) age at surgery; (B) type of gastrectomy; (C) ELNs; (D) tumor size. ELNs, examined lymph nodes.

(Table 3, Figure 6). This was also true for patients in the SEER cohort stratified according to sex, age at surgery, tumor size, tumor location, and Lauren classification $(\mathrm{P}<0.05)$ : female (HR 2.778, $\mathrm{P}=0.037$ ), male (HR 8.667, $\mathrm{P}=0.035)$, age $>60$ years $(\mathrm{HR} 3.886, \mathrm{P}=0.011$ ), tumor $>4 \mathrm{~cm}$ (HR 3.211, $\mathrm{P}=0.015)$, lower third (HR 3.536, $\mathrm{P}=0.026),>2 / 3$ stomach (HR 4.203, $\mathrm{P}=0.035)$, intestinal GC (HR 7.768, $\mathrm{P}=0.015$ ), and diffuse GC (HR 4.158, $\mathrm{P}=0.034$ (Table 4, Figure 7).

These results demonstrated that $>31$ ELNs was a prerequisite for accurate evaluation of prognosis in patients with node-negative stage III GC.

\section{Stage migration in patients with stage III node-negative GC}

All 84 stage III node-negative (pN0 stage) patients and 437 patients with $\mathrm{pN} 1$ stage from the Chinese institutions were included in the stage migration analysis using the log-rank test. There was no significant difference in survival between pT4bN0M0 and pT4bN1M0 stage GC among patients with $<31$ ELNs (Figure 8A, $\mathrm{P}=0.116$ ). Conversely, there was a significant survival difference between these stages in patients with $>31 \mathrm{ELNs}$ (Figure $8 B, \mathrm{P}=0.038$ ), indicating that $>31$ ELNs might result in stage migration of patients with pT4bN0M0 GC. Therefore, $>31$ ELNs could be considered as an indispensable prerequisite for guaranteeing accurate pathological staging and precise prognostic evaluation in patients with node-negative stage III GC.

\section{Nomogram analysis of patients with node-negative GC}

To predict OS among node-negative patients with stage III GC, we further applied the independent risk factors identified by multivariate analysis to nomogram analysis in Chinese cohort (Figure 9).

\section{Discussion}

The number of ELNs is considered an independent prognostic factor in multiple cancers (21-23). Previous studies showed that a greater number of ELNs was associated with more precise nodal staging, which could largely explain the survival association $(24,25)$. Furthermore, we previously demonstrated that an insufficient number of ELNs might be a potential risk factor for postoperative recurrence in patients with GC, including node-negative patients (26-28). Although the AJCC recommends a minimum of 15 ELNs, there is currently no consensus on the association between ELN count and survival or on the threshold number of ELNs to address both stage migration and long-term survival in GC, especially stage III GC.

In this study, we analyzed the association between ELN 
Table 2 Clinicopathologic variables and univariate analysis of the SEER cohort of node-negative stage III gastric cancer patients

\begin{tabular}{|c|c|c|c|c|c|c|}
\hline Population & $\begin{array}{c}\text { No. of } \\
\text { patients }\end{array}$ & $\begin{array}{l}\text { 3-year } \\
\text { OS (\%) }\end{array}$ & $\begin{array}{l}\text { 5-year } \\
\text { OS (\%) }\end{array}$ & $\frac{\text { Univariate analysis }}{\mathrm{P} \text { value }}$ & \multicolumn{2}{|c|}{ Multivariate analysis } \\
\hline Gender & & & & 0.913 & & \\
\hline Female & 76 & 35.0 & 29.8 & & & \\
\hline Male & 120 & 36.8 & 30.1 & & & \\
\hline$\leq 60$ & 66 & 45.5 & 40.3 & & 1 (Ref.) & \\
\hline$>60$ & 130 & 30.8 & 26.9 & & $1.584(1.105-2.269)$ & \\
\hline Tumor size, $\mathrm{cm}$ & & & & 0.184 & & 0.591 \\
\hline$\leq 4$ & 42 & 38.1 & 33.2 & & 1 (Ref.) & \\
\hline Tumor location & & & & 0.570 & & \\
\hline Upper third & 23 & 26.1 & 21.7 & & & \\
\hline Middle third & 24 & 45.8 & 41.7 & & & \\
\hline Lower third & 86 & 36.0 & 30.9 & & & \\
\hline$>2 / 3$ stomach & 31 & 32.3 & 22.6 & & & \\
\hline Unknown & 32 & 37.5 & 33.8 & & & \\
\hline Lauren classification & & & & 0.120 & & 0.216 \\
\hline Intestinal & 65 & 41.5 & 34.9 & & 1 (Ref.) & \\
\hline
\end{tabular}

No., number; OS, overall survival; HR, hazard ratio; 95\% Cl, 95\% confidence interval; Ref, reference; ELNs, examined lymph nodes; SEER, Surveillance, Epidemiology, and End Results..
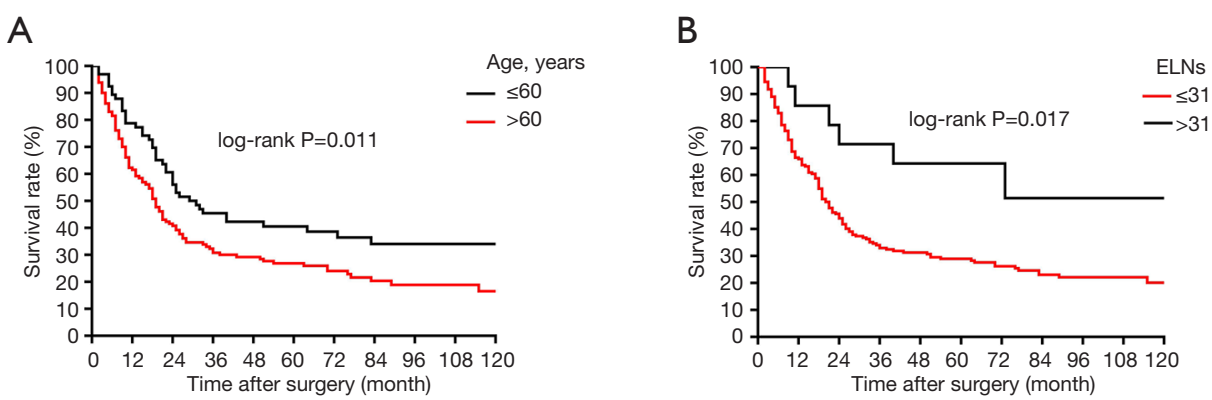

Figure 5 Survival curves of patients in SEER cohort according to subgroups: (A) age at surgery; (B) ELNs. ELNs, examined lymph nodes; SEER, Surveillance, Epidemiology, and End Results. 
Table 3 Subgroup analyses of optimal cutoff value in different population sets of Chinese cohort

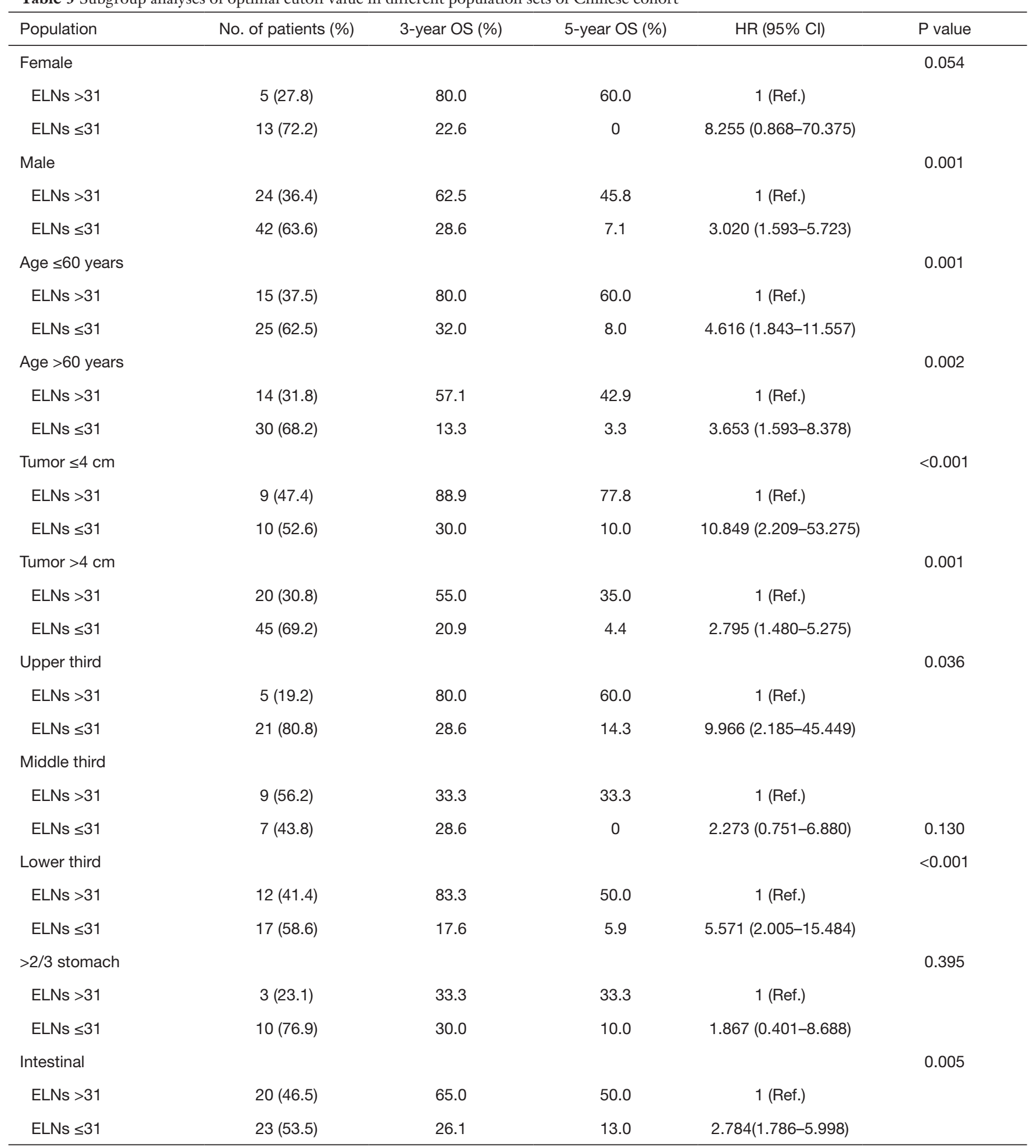

Table 3 (continued) 
Table 3 (continued)

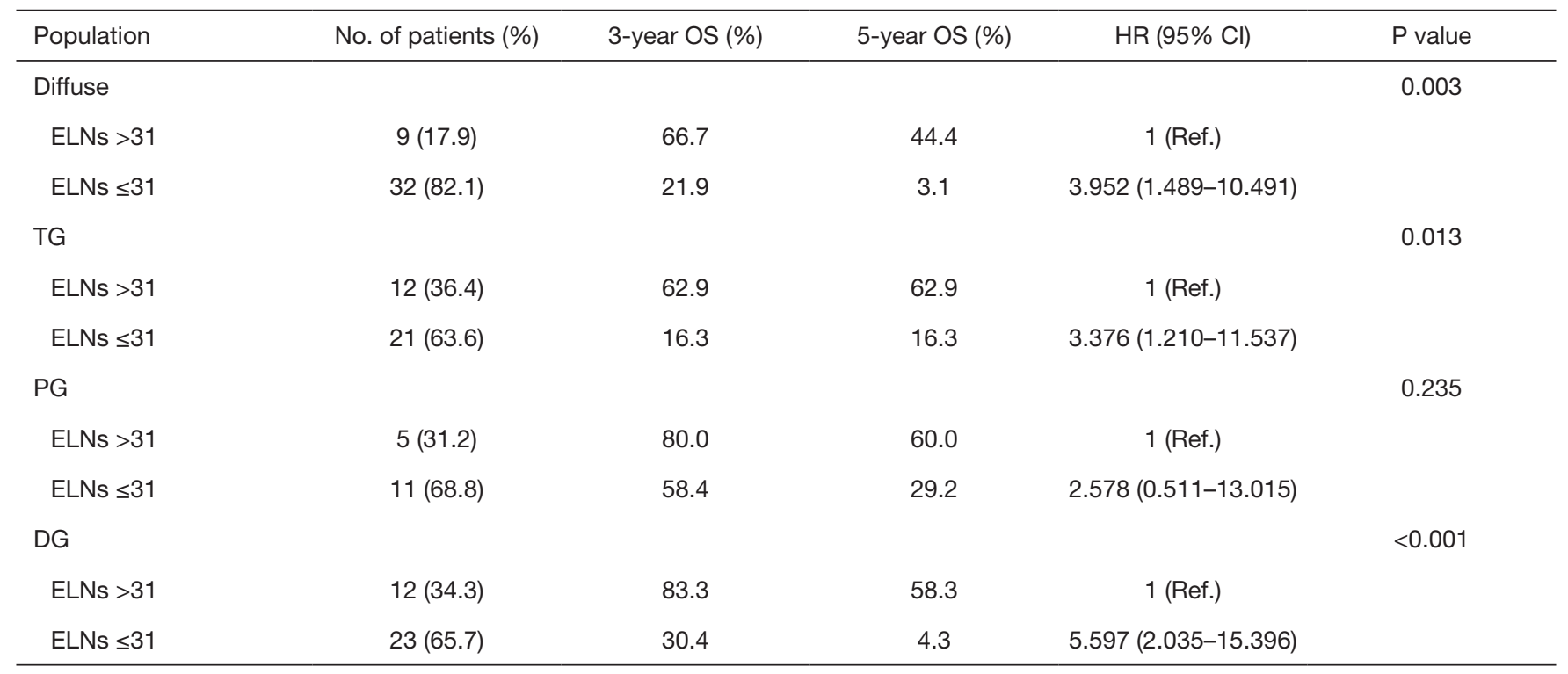

No., number; OS, overall survival; HR, hazard ratio; 95\% Cl, 95\% confidence interval; ELNs, examined lymph nodes; Ref, reference; TG; total gastrectomy; PG, proximal gastrectomy; DG, distal gastrectomy.

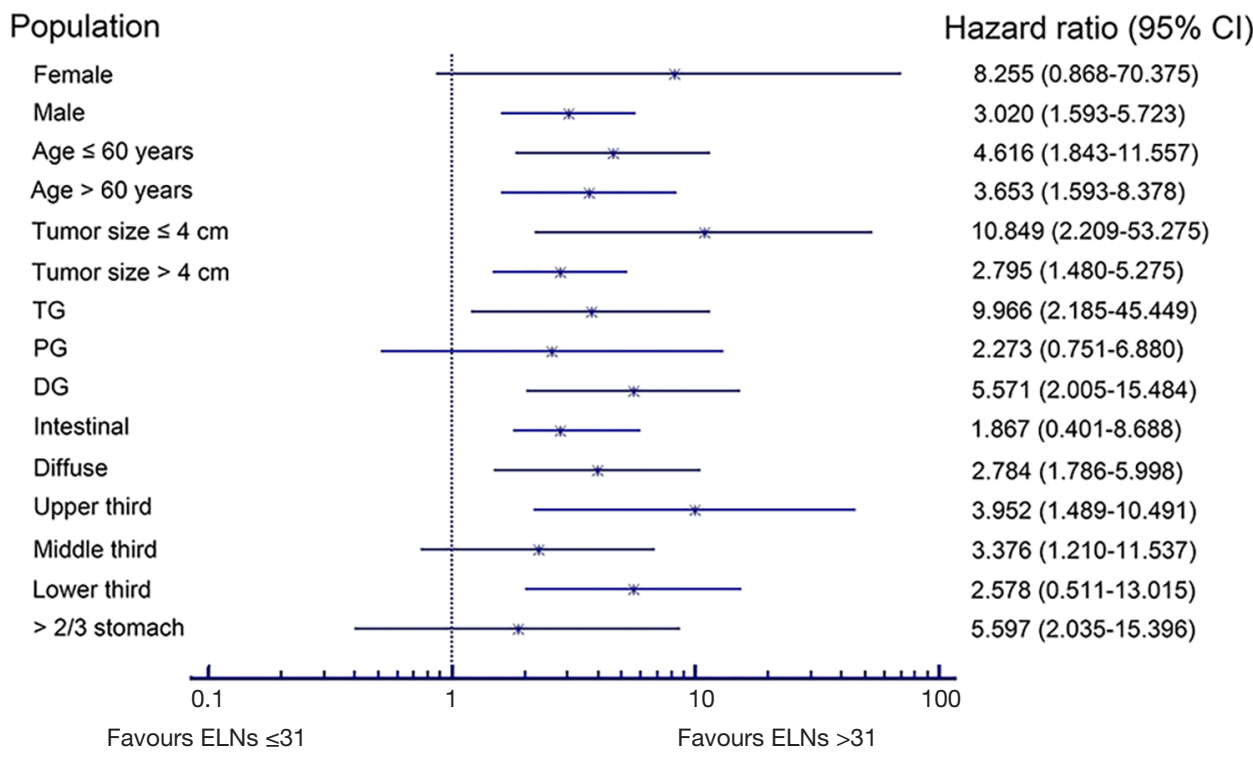

Figure 6 Forest plot of overall survival (OS) of the Chinese training cohort. Subgroup analyses of OS of the Chinese training cohort were performed using patient baseline characteristics.

number and long-term survival of patients with resected node-negative stage III GC. All the ELNs were negative, which was previously identified as a factor positively associated with prognosis in GC (29-32). The current results demonstrated a significant association between the number of ELNs and the prognosis of patients with nodenegative stage III GC after gastrectomy with systemic lymphadenectomy. An optimal cut-off value of 31 ELNs was determined in the Chinese training cohort and validated in the SEER cohort, with good ability to discriminate survival 
Table 4 Subgroup analyses of optimal cutoff value in different population sets of SEER cohort

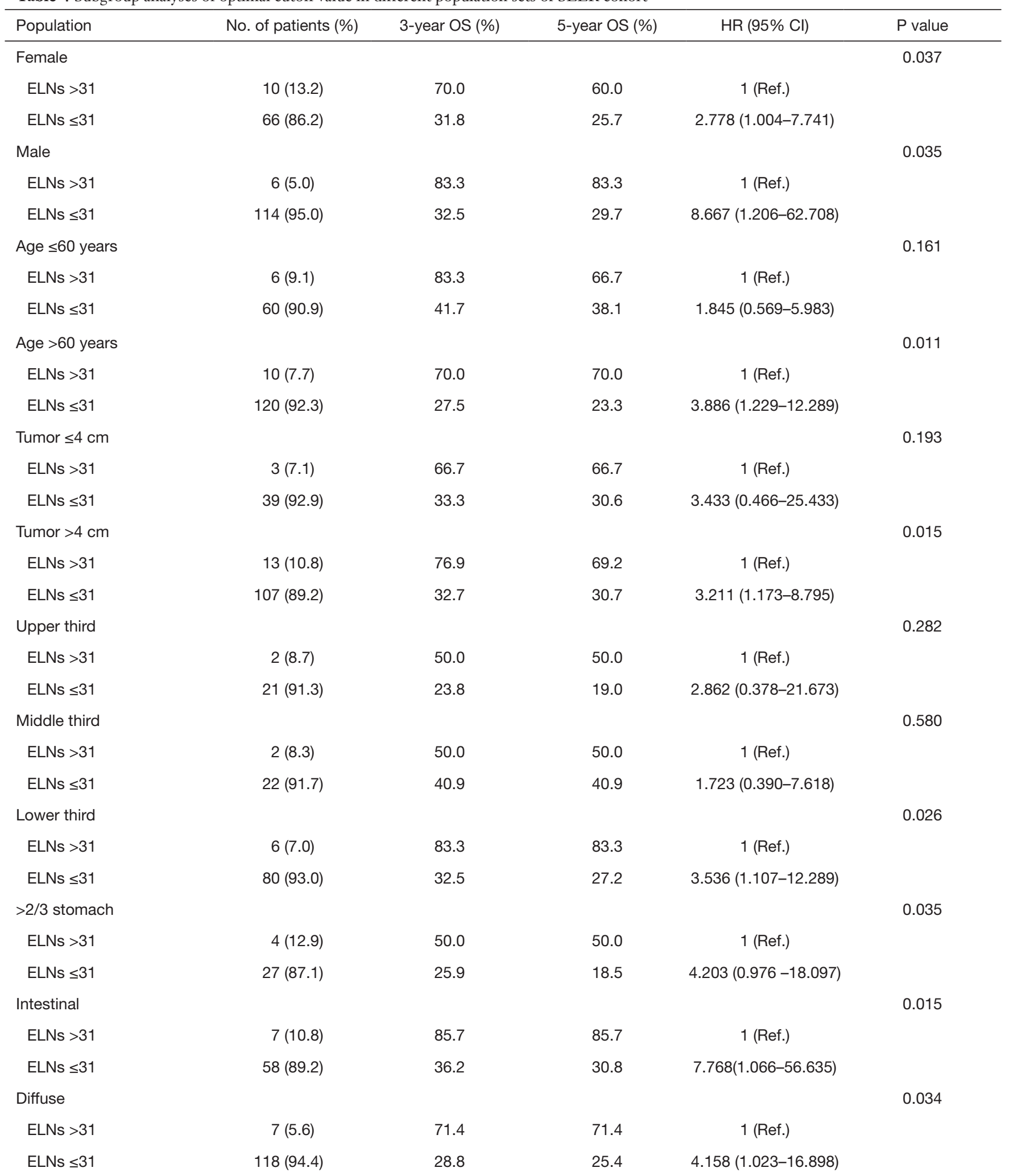

No., number; OS, overall survival; HR, hazard ratio; 95\% CI, 95\% confidence interval; ELNs, examined lymph nodes; Ref, reference; TG; total gastrectomy; PG, proximal gastrectomy; DG, distal gastrectomy; SEER, Surveillance, Epidemiology, and End Results. 


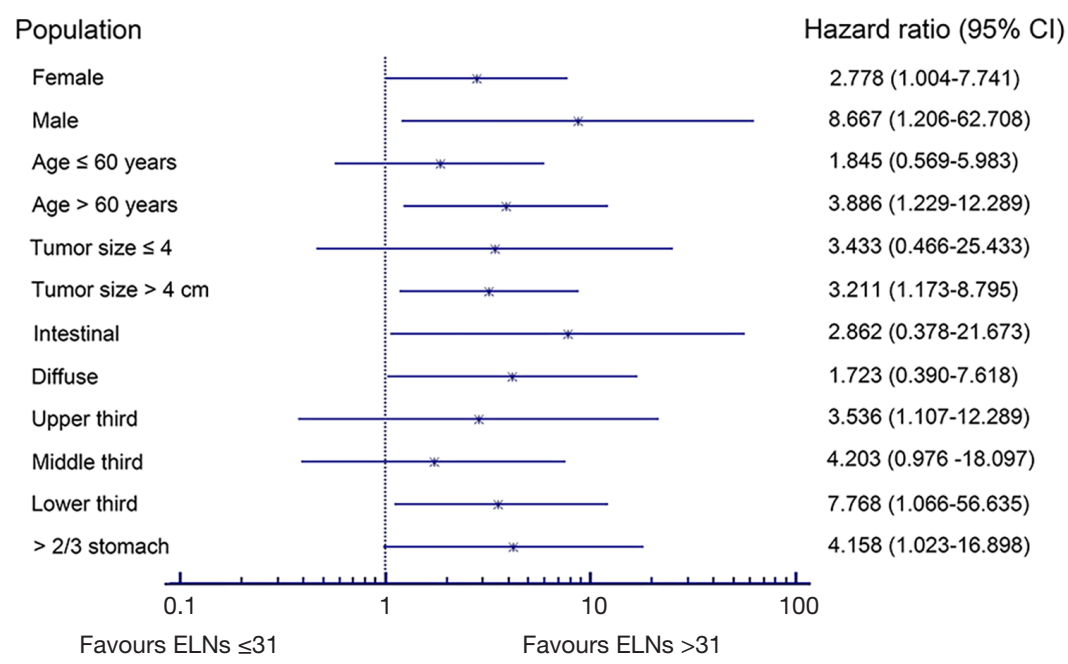

Figure 7 Forest plot of overall survival (OS) of the Surveillance, Epidemiology, and End Results (SEER) database cohort. Subgroup analyses of OS of the SEER cohort were performed using patient baseline characteristics.
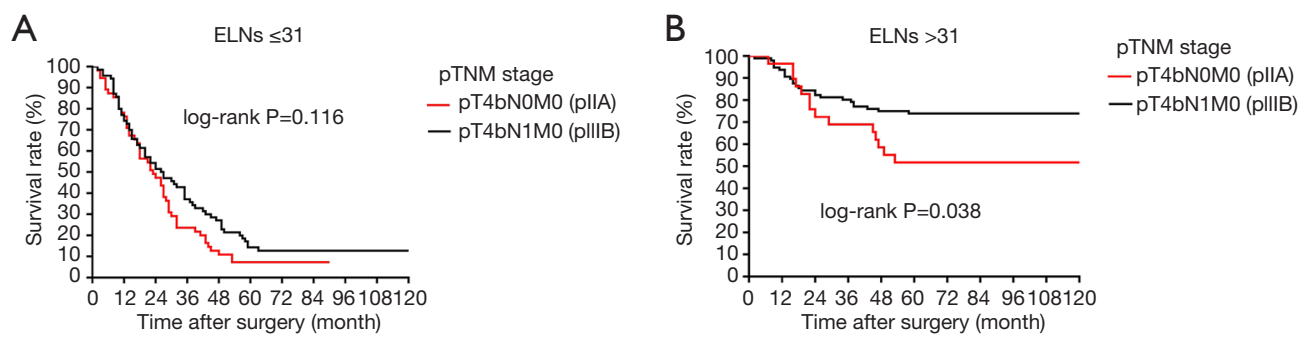

Figure 8 (A) Survival curves of the different subgroups of patients according to pTNM stage when the ELNs $\leq 31$; (B) survival curves of the different subgroups of patients according to pTNM stage when the ELNs $>31$. ELNs, examined lymph nodes.

probabilities. The number of ELNs $(\leq 31$ versus $>31)$ was also shown to be an independent prognostic factor in nodenegative patients. This study further indicated that patients with node-negative GC and $\leq 31$ ELNs had significantly poorer OS rates and a higher risk of mortality than patients with $>31$ ELNs in both cohorts, irrespective of multiple clinicopathological factors.

Examination of more LNs is known to reduce the risk of undetected positive LNs, allowing the more thorough elimination of remnants and proper delivery of adjuvant chemotherapy to improve long-term survival, particularly in node-negative patients. Harvesting of a lower number of LNs increases the likelihood of missing positive nodal disease, potentially resulting in understaging and inappropriate patient selection for adjuvant systemic therapy. However, there is no consensus on the mechanisms responsible for the better survival associated with a higher number of ELNs.

Previous studies showed that the better survival of patients with a higher number of ELNs may be secondary to stage migration and better patient selection for adjuvant systemic therapy (32-34). Zhao et al. (35) recommended $\geq 25$ ELNs in patients with advanced GC and demonstrated that the AJCC recommendation of $\geq 15$ ELNs was insufficient for determining the $\mathrm{N}$ stage. Woo et al. (36) further pointed out that $\geq 29$ ELNs was associated with a maximum survival advantage in patients with GC undergoing surgery, using the SEER database and a Korean database of 25,289 patients with GC. A higher LN harvest can potentially prevent understaging by shifting patients from a lower to a higher TNM stage. Furthermore, the number of ELNs reflects the actual number of LNs removed surgically as well as the number of LNs identified and examined during macroscopic and microscopic pathological analyses. The 


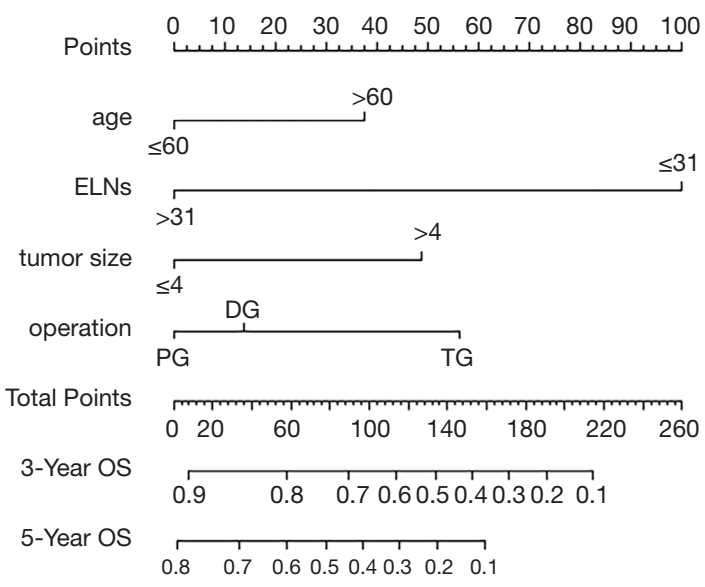

Figure 9 A nomogram established for predicting 3- and 5-year survival. The nomogram was developed in the training group by incorporating the following parameters: age (years), ELNs, tumor size $(\mathrm{cm})$, type of operation. ELNs, examined lymph nodes.

number of ELNs after surgery could be affected by an insufficient lymph node dissection or destruction of $\mathrm{LN}$ integrity during surgery. Furthermore, surgical procedures, assessments, and enumeration of LNs vary among regions, surgeons, laboratories, and pathologists. Some researchers have pointed out that $\mathrm{LN}$ harvest may also be a proxy for the quality of the surgical resection, accounting for the better outcomes (37). Postoperative examination of an inadequate number of retrieved LNs may also result in inadequate staging and subsequent treatment $(28,33)$. In comparison to pathologists, surgeons are more familiar with the anatomical location of LNs, the direction of LN drainage, and the location of LN metastases corresponding to different resection areas. Submission of ELNs by skilled surgeons may thus reduce human error in the number of ELNs.

Alternatively, a higher number of LNs indicates a stronger immune reaction to the tumor, which is a wellknown predictor of better prognosis. Large amounts of perigastric lymphoid tissue may be associated with greater barriers to defense against metastasis and robust tumor immunity, and patients with fewer retrieved LNs may thus have lower barriers to defense against metastasis and diminished tumor immunity. LN size and morphology are modified by immune responses (38-40). Distension and prominence of the lymphatic sinusoids may be related to the host immune response against neoplastic cell products. Once the immune system detects a tumor, the antitumor immune response causes $\mathrm{LN}$ enlargement, thus improving their detectability by surgeons and pathologists. Patients with more ELNs may thus have a better prognosis due to a more robust immune response. The current study was based on real-world patient data, and we cannot rule out the possibility of a positive correlation between the immune response and ELNs.

This study had certain limitations. Our training cohort was based on a Chinese population database and the applicability of the results in terms of the optimal number of ELNs to populations from other countries has yet to be validated. Furthermore, the treatment protocol for locally advanced GC of the same TNM category differs between Asian and Western cancer centers, and may explain the lower 5-year OS rate in the SEER cohort compared with the Chinese cohort. Neoadjuvant therapies followed by radical resection (including D1 or D1+ lymphadenectomy) are conventionally used in Western countries, while radical surgery (D2 or D2 + lymphadenectomy) followed by adjuvant therapy is primarily considered in Asian cancer centers. We therefore used the SEER database for external validation of the proposed optimal number of ELNs. In addition, to the best of our knowledge the sample size for the training cohort was based on the three highestvolume GC centers across North and South China and was the largest among all such previous studies. This further supports the reliability of the present results. However, although the patients came from the three highest-volume GC centers across North and South China, the sample size was still relatively small and further studies with larger sample sizes are required to confirm the present findings.

\section{Conclusions}

Using large cohorts of patients from multiple Chinese institutions and the SEER database, we demonstrated that the number of ELNs was an independent prognostic factor in patients with stage III node-negative GC. More than 31 ELNs was associated with better survival and is therefore proposed as recommended practice for patients with stage III GC, especially node-negative patients. This number of ELNs demonstrated superior stratification and prognosis prediction abilities, suggesting high potential for clinical application in different populations.

\section{Acknowledgments}

Funding: This work was supported in part by grants from 
the Programs of National Natural Science Foundation of China (No. 81572372, No. 81172080, No. 81201773, No. 81572372), National Key Research and Development Program (MOST-2016YFC1303202), National Precision Medicine Research Program (2017YFC0908300), the Application Foundation and Advanced Technology Program of Tianjin Municipal Science and Technology Commission (15JCYBJC24800) and the National Key Clinical Specialist Construction Programs of China (2013-544).

\section{Footnote}

Reporting Checklist: The authors have completed the STROBE reporting checklist. Available at http://dx.doi. org/10.21037/atm-20-1358a

Data Sharing Statement: Available at http://dx.doi. org/10.21037/atm-20-1358a

Conflicts of Interest: All authors have completed the ICMJE uniform disclosure form (available at http://dx.doi. org/10.21037/atm-20-1358a). The authors have no conflicts of interest to declare.

Ethical Statement: The authors are accountable for all aspects of the work in ensuring that questions related to the accuracy or integrity of any part of the work are appropriately investigated and resolved. The study was conducted in accordance with the Declaration of Helsinki (as revised in 2013). Ethical approval was obtained from the institutional review board of the Tianjin Medical University Cancer Institute \& Hospital (No: bc2018037). Individual consent for this retrospective analysis was waived.

Open Access Statement: This is an Open Access article distributed in accordance with the Creative Commons Attribution-NonCommercial-NoDerivs 4.0 International License (CC BY-NC-ND 4.0), which permits the noncommercial replication and distribution of the article with the strict proviso that no changes or edits are made and the original work is properly cited (including links to both the formal publication through the relevant DOI and the license). See: https://creativecommons.org/licenses/by-nc-nd/4.0/.

\section{References}

1. Global Burden of Disease Cancer Collaboration, Fitzmaurice C, Dicker D, et al. The Global Burden of
Cancer 2013. JAMA Oncol 2015;1:505-27.

2. Leung WK, Wu MS, Kakugawa Y, et al. Screening for gastric cancer in Asia: current evidence and practice. Lancet Oncol 2008;9:279-87.

3. González CA, Agudo A. Carcinogenesis, prevention and early detection of gastric cancer: where we are and where we should go. Int J Cancer 2012;130:745-53.

4. Van Cutsem E, Sagaert X, Topal B, et al. Gastric cancer. Lancet 2016;388:2654-64.

5. Foo M, Leong T. Adjuvant therapy for gastric cancer: current and future directions. World J Gastroenterol 2014;20:13718-27.

6. Kanda M, Kodera Y, Sakamoto J. Updated evidence on adjuvant treatments for gastric cancer. Expert Rev Gastroenterol Hepatol 2015;9:1549-60.

7. Valsangkar NP, Bush DM, Michaelson JS, et al. N0/ N1, PNL, or LNR? The effect of lymph node number on accurate survival prediction in pancreatic ductal adenocarcinoma. J Gastrointest Surg 2013;17:257-66.

8. Macalindong SS, Kim KH, Nam BH, et al. Effect of total number of harvested lymph nodes on survival outcomes after curative resection for gastric adenocarcinoma: findings from an eastern high-volume gastric cancer center. BMC Cancer 2018;18:73.

9. Sun Z, Zhu GL, Lu C, et al. The impact of N-ratio in minimizing stage migration phenomenon in gastric cancer patients with insufficient number or level of lymph node retrieved: results from a Chinese mono-institutional study in 2159 patients. Ann Oncol 2009;20:897-905.

10. Kim JJ, Song KY, Hur H, et al. Lymph node micrometastasis in node-negative early gastric cancer. Eur J Surg Oncol 2009;35:409-14.

11. Biffi R, Botteri E, Cenciarelli S, et al. Impact on survival of the number of lymph nodes removed in patients with node-negative gastric cancer submitted to extended lymph node dissection. Eur J Surg Oncol 2011;37:305-11.

12. He H, Shen Z, Wang X, et al. Survival benefit of greater number of lymph nodes dissection for advanced node-negative gastric cancer patients following radical gastrectomy. Jpn J Clin Oncol 2016;46:63-70.

13. Li B, Li Y, Wang W, et al. Incorporation of N0 stage with insufficient numbers of lymph nodes into N1 stage in the seventh edition of the TNM classification improves prediction of prognosis in gastric cancer: results of a single-institution study of 1258 Chinese patients. Ann Surg Oncol 2016;23:142-8.

14. von Elm E, Altman DG, Egger M, et al. The Strengthening the Reporting of Observational Studies 
in Epidemiology (STROBE) statement: guidelines for reporting observational studies. PLoS Med 2007;16;4:e296.

15. Ndebele P. The Declaration of Helsinki, 50 years later. JAMA 2013;310:2145-6.

16. Jaehne J, Meyer HJ, Maschek H, et al. Lymphadenectomy in Gastric Carcinoma. A Prospective and Prognostic Study. Arch Surg 1992;127:290-4.

17. Nakajima T. Gastric cancer treatment guidelines in Japan. Gastric Cancer 2002;5:1-5.

18. Liang $\mathrm{Y}, \mathrm{Wu} \mathrm{L}$, Liu L, et al. Impact of extranodal tumor deposits on prognosis and $\mathrm{N}$ stage in gastric cancer. Surgery 2019;166:305-13.

19. National Cancer Institute. Surveillance, Epidemiology, and End Results (SEER) Program (www.seer.cancer.gov) Research Data (1973-2015). Bethesda, MD: National Cancer Institute, 2018.

20. Feinstein AR, Sosin DM, Wells CK. The Will Rogers phenomenon. Stage migration and new diagnostic techniques as a source of misleading statistics for survival in cancer. N Engl J Med 1985;312:1604-8.

21. Liang W, He J, Shen Y, et al. Impact of Examined Lymph Node Count on Precise Staging and Long-Term Survival of Resected Non-Small-Cell Lung Cancer: A Population Study of the US SEER Database and a Chinese MultiInstitutional Registry. J Clin Oncol 2017;35:1162-70.

22. Huang L, Jansen L, Balavarca Y, et al. Significance of Examined Lymph Node Number in Accurate Staging and Long-term Survival in Resected Stage I-II Pancreatic Cancer-More is Better? A Large International Population-based Cohort Study. Ann Surg 2019. [Epub ahead of print].

23. Johnson PM, Porter GA, Ricciardi R, et al. Increasing Negative Lymph Node Count Is Independently Associated With Improved Long-Term Survival in Stage IIIB and IIIC Colon Cancer. J Clin Oncol 2006;24:3570-5.

24. Hall MD, Schultheiss TE, Smith DD, et al. Impact of total lymph node count on staging and survival after neoadjuvant chemoradiation therapy for rectal cancer. Ann Surg Oncol 2015;22:S580-7.

25. Li F, Hong X, Hou L, et al. A greater number of dissected lymph nodes is associated with more favorable outcomes in bladder cancer treated by radical cystectomy: a metaanalysis. Oncotarget 2016;7:61284-94.

26. Deng J, Yamashita H, Seto Y, et al. Increasing the Number of Examined Lymph Nodes is a Prerequisite for Improvement in the Accurate Evaluation of Overall Survival of Node-Negative Gastric Cancer Patients. Ann
Surg Oncol 2017;24:745-53.

27. Jiao XG, Deng JY, Zhang RP, et al. Prognostic value of number of examined lymph nodes in patients with node-negative gastric cancer. World J Gastroenterol 2014;20:3640-8.

28. Deng J, Liu J, Wang W, et al. Validation of clinical significance of examined lymph node count for accurate prognostic evaluation of gastric cancer for the eighth edition of the American Joint Committee on Cancer (AJCC) TNM staging system. Chin J Cancer Res 2018;30:477-91.

29. Deng J, Zhang R, Zhang L, et al. Negative node count improvement prognostic prediction of the seventh edition of the TNM classification for gastric cancer. PLoS One 2013;8:e80082.

30. Deng J, Liang H, Wang DC, et al. Enhancement the prediction of postoperative survival in gastric cancer by combining the negative lymph node count with ratio between positive and examined lymph nodes. Ann Surg Oncol 2010;17:1043-51.

31. Deng J, Sun D, Pan Y, et al. Ratio between negative and positive lymph nodes is suitable for evaluation the prognosis of gastric cancer patients with positive node metastasis following curative surgery. PLoS One 2012;7:e43925.

32. Deng J, Zhang R, Wu LL, et al. Superiority of the ratio between negative and positive lymph nodes for predicting the prognosis for patients with gastric cancer. Ann Surg Oncol 2015;22:1258-66.

33. Smith DD, Schwarz RR, Schwarz RE. Impact of Total lymph node count on staging and survival after Gastrectomy for gastric cancer: data from large USpopulation database. J Clin Oncol 2005;23:7114-24.

34. Bouvier AM, Haas O, Piard F, et al. How many nodes must be examined to accurately stage gastric carcinomas? Results from a population-based study. Cancer 2002;94:2862-6.

35. Zhao B, Zhang J, Chen X, et al. The retrieval of at least 25 lymph nodes should be essential for advanced gastric cancer patients with lymph node metastasis: a retrospective analysis of single-institution database study design: Cohort study. Int J Surg 2017;48:291-9.

36. Woo Y, Goldner B, Ituarte P, et al. Lymphadenectomy with optimum of 29 lymph nodes retrieved associated with improved survival in advanced gastric cancer: a 25,000-Patient International Database Study. J Am Coll Surg 2017;224:546-55.

37. Hayashi S, Kanda M, Ito S, et al. Number of retrieved lymph nodes is an independent prognostic factor after 
total gastrectomy for patients with stage III gastric cancer: propensity score matching analysis of a multi-institution dataset. Gastric Cancer 2019;22:853-63.

38. Zhou J, Zhang WW, Wu SG, et al. The impact of examined lymph node count on survival in squamous cell carcinoma and adenocarcinoma of the uterine cervix. Cancer Manag Res 2017;9:315-22.

39. Degiuli M, Arolfo S, Evangelista A, et al. Number of lymph nodes assessed has no prognostic impact in node-

Cite this article as: Zhang N, Bai H, Deng J, Wang W, Sun Z, Wang Z, Xu H, Zhou Z, Liang H. Impact of examined lymph node count on staging and long-term survival of patients with node-negative stage III gastric cancer: a retrospective study using a Chinese multi-institutional registry with Surveillance, Epidemiology, and End Results (SEER) data validation. Ann Transl Med 2020;8(17):1075. doi: 10.21037/atm-20-1358a negative rectal cancers after neoadjuvant therapy. Results of the "Italian Society of Surgical Oncology (S.I.C.O.) Colorectal Cancer Network" (SICO-CCN) multicentre collaborative study. Eur J Surg Oncol 2018;44:1233-40.

40. He WZ, Xie QK, Hu WM, et al. An increased number of negative lymph nodes is associated with a higher immune response and longer survival in colon cancer patients. Cancer Manag Res 2018;10:1597-604. 